\title{
A Retrospective observational study on dietary factors influencing Urolithiasis
}

\author{
Dr.P Saravana kumar ${ }^{1}$, Gurijala Sai mounika ${ }^{1}$, Konala raja prabhu sujith ${ }^{1}$, Rajulapati Tejaswini ${ }^{1}$ \\ 1, Department of urology, Lalitha super speciality hospital, Guntur. \\ 1,1,1 Department of pharmacy practice, AM Reddy memorial college of pharmacy, Narasaraopet, Guntur (Dst), Andhra Pradesh. \\ DOI: 10.29322/IJSRP.10.12.2020.p10814 \\ http://dx.doi.org/10.29322/IJSRP.10.12.2020.p10814
}

\begin{abstract}
Urolithiasis is defined as the multi factorial disorder which involves the process of forming stones anywhere in the urinary tract (kidney, ureter, and bladder).Out of many factors diet plays a major role in forming urolithiasis. If left untreated it can lead to severe complications. Dietary and life style modifications can make a change.

Methodology: This study is a retrospective observational study and the data is collected from patients who are diagnosed with urolithiasis in a tertiary care hospital which includes 160 patients and their data is collected using food frequency questionnaire form which is validated by the senior urologist.

Results: Among the 160 patients, 103 patients were males and 57 females. The participants were belonged to the age group of 19 to 88 ( $p$ value $<0.001$ ).As per our study the major risk factors are high intake of oxalate rich food, high animal protein diet, less fluid intake and more salt intake will increase the risk of urolithiasis. It was found that less fluid intake and high salt intake will influence the risk of urolithiasis $(\mathrm{p}<0.0001)$.Moreover the patients with urolithiasis consume high oxalate rich food and more animal protein intake $(68.25 \%)$.

Conclusion: The results show that there is a clear association between dietary factors and urolithiasis occurrence in males and females. From our study we conclude that less fluid intake, high oxalate foods, and high animal protein diet, high dietary salt will influence the formation of urolithiasis.
\end{abstract}

Index Terms- Kidney stones, Dietary risk factors, Urinary tract stones, Less fluid intake, Personalized diet plan.

\section{INTRODUCTION}

$\mathrm{U}$ rolithiasis is defined as the process of forming stones in the kidney,bladder and urethra (urinary tract). ${ }^{[1]}$ The overall probability of forming caliculi differ in various parts of the world and is estimated as 1-5\% in Asia, 5-9\% in Europe, 13\% in North America and the recurrence rate of renal stones about $75 \%$ in 20 years span . It occurs both in men and women but the risk is generally high in men and is becoming more common in young women. ${ }^{[2]}$

\section{TYPES OF CALCULI}

Most commonly occurred renal caliculi are: Calcium oxalate, uric acid, Struvite and cystine stones. Risk factors of calcium oxalate stones include dehydration, hypercalciuria, hyper uricosuria. ${ }^{[3]}$ Risk factors of uric acid stones include consumption of meat, chicken and sea food these increases uric acid production thus leads to uric acid stones. ${ }^{[4]}$. Risk factors of Struvite stones are of from infections. The most common being Proteus mirabilis and less common pathogens include Klebsiella pneumonia. ${ }^{[5]}$ and cystine stones occurred due to a genetic disorder. which is an autosomal recessive disorder caused by a defect in the rBAT gene on chromosome 2 resulting in impaired renal tubular absorption of cysteine or leaking cysteine into urine. ${ }^{[6]}$

\section{Clinical PRESENTATIONS:}

Symptoms based on sites:

Renal caliculi: Loin pain, vomiting.

Ureteric caliculi: groin pain, fever, chills, and dysuria.

Bladder caliculi: Supra-pubic pain, dysuria, haematuria and may also present with UTI Symptoms (nocturia, urgency, nausea).

\section{RISK FACTORS:}

Risk factors are of mainly hereditary,(Idiopathic hypercalciuria, hyperoxalosis, cystinuria: Dent's disease),disease related (Medullary sponge kidney,Poly cystic kidney disease, (10\% develop stones,), renal tubular acidosis) dietary(high sodium diet, oxalate rich foods, animal protein, high protein weight loss diet),dehydration (low urine volume),stress, heat, water loss, sweating, and some drugs like furosemide, Indinavir, allopurinol etc. ${ }^{[7]}$

\section{AIM}

The aim of the study was to assess the dietary factors influencing urolithiasis.

\subsection{Objectives:}

- To identify and evaluate the occurrence of urolithiasis in the out patients and In-patients in a tertiary care hospital. The data is collected from patients who were diagnosed with urolithiasis at tertiary care Hospital.

- To collect the data using food frequency questionarrie form which includes their dietary habits which results in occurrence of urolithiasis.

- To Counsel the patients regarding their life style modifications to prevent the recurrence of urolithiasis. 


\subsection{Study design:}

The current study is a retrospective observational study performed by using food frequency questionarrie form for the assessment of dietary factors influencing urolithiasis.

\subsection{Study site:}

The study was carried out in tertiary care hospital of Guntur district, Andhra Pradesh, INDIA.

\subsection{Study Period:}

The retrospective observational study was conducted over a period of six months from October 2019 to march 2020.

\subsection{Study Population \& Sampling:}

Sample of 160 subjects were taken in our study, knowledge of the cause of a disease is one of the most important factors in the control of the disease, 'Urolithiasis is occured majorily due to lack of awareness on the dietary habits

\subsection{Study Criteria :}

Inclusion criteria:

1. Population who are in between 19 years to 89 years and are willing to participate.

Fig 1 Distribution of male and females

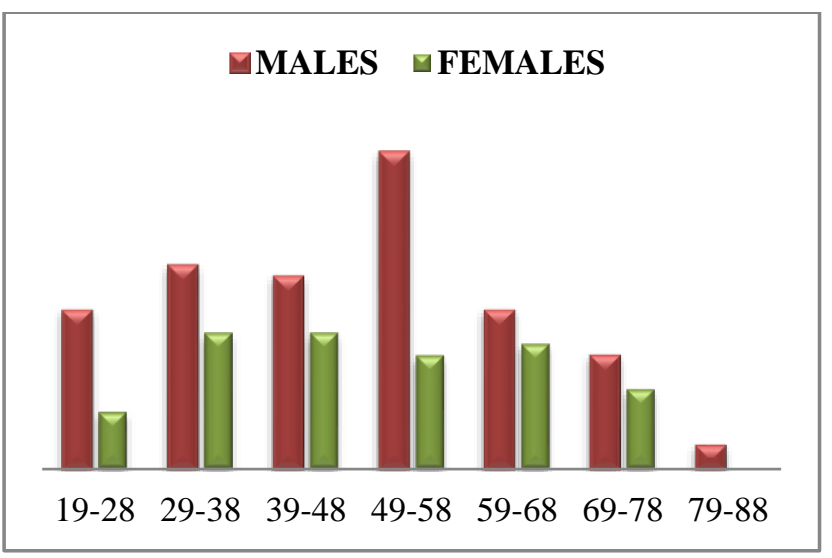

Fig 1 represents the distribution of males and females. Among the ages 19-88 males are of more diagnosed with urolithiasis compared to females. There is a significant relationship with $p$ value $<0.001$ with a 0.05 level of significance and the standard deviation was found to be 10.69 and Fig 2
2. Both male and female patients of both Outpatients and Inpatients are included.

Exclusion criteria:

1. The patients below $19 y$ yrs and above $89 y$ rs.

2. The patients who were non respondent to the study.

3. The pregnant ladies, lactating women and post menopausal women.

4.The patients using calcium supplements and vitamin-d and vit-c supplements

5.The patients having metabolic disorders i,e hyperparathyroidism, gout, hyper calciuria.

\subsection{Results \& discussion:}

A total of 160 patients were included in the study. Among them 103 are males and 57 are females. The age group which are more prone to urolithiasis are of from 49-58. The mean age was found to be 48.24. The data collected was significant with a $\mathrm{p}$ value $<0.0001$ at 0.05 level of significance and the standard deviation is 10.68. Figure 1 represents the distribution of male and females. Among the total population

Fig 2clinical presentations

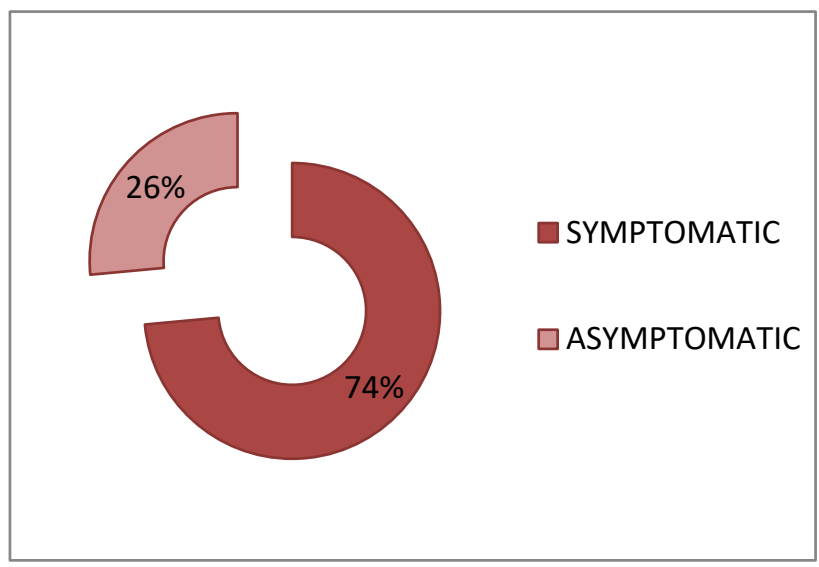

represents clinical presentations. Symptomatic includes patients with loin pain, groin pain and flank pain haematuria, dysuria. In our study $76 \%$ are symptomatic, About $26 \%$ are asymptomatic, includes patients with no symptoms and unaware of calculi which is diagnosed in their ultra sound scan reports. 
Fig 3 Risk factors

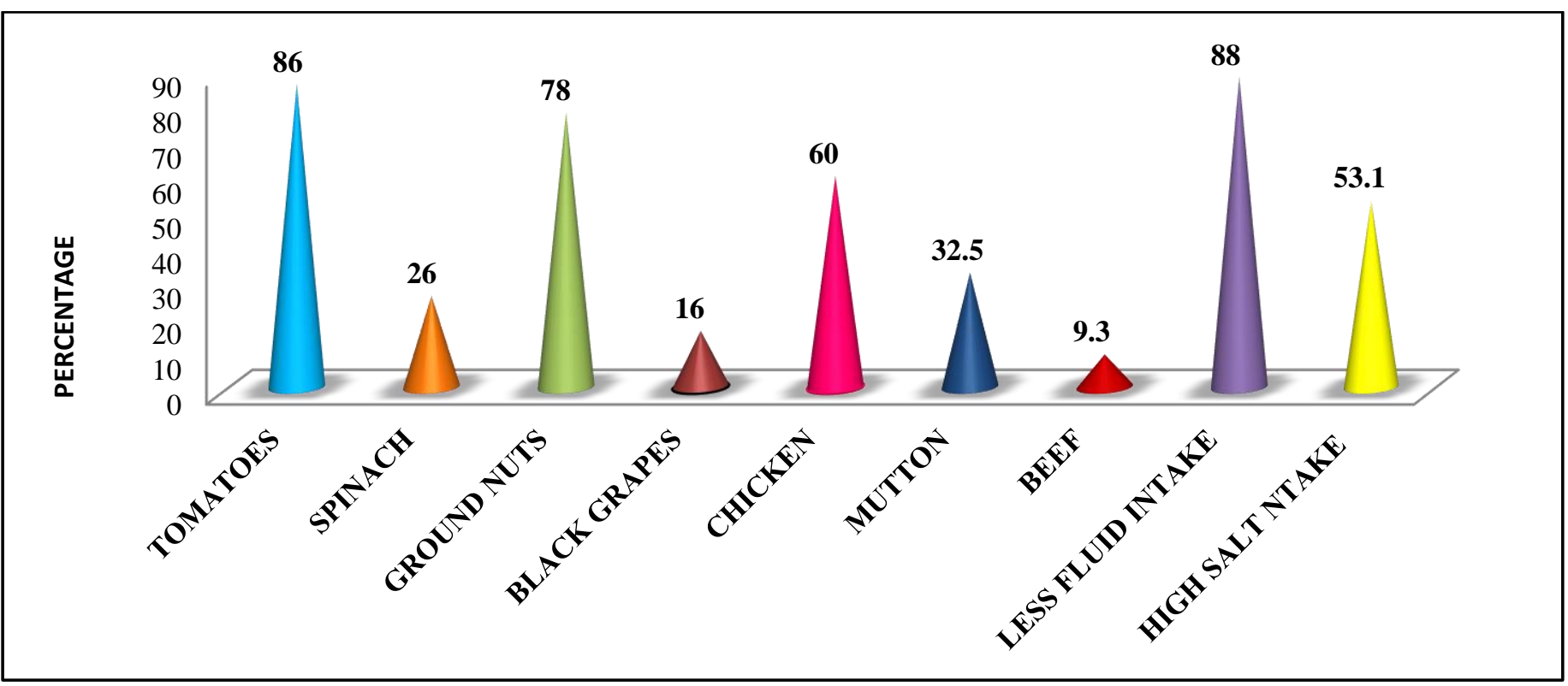

Fig 3 represents risk factors. In our study the major dietary risk factors of urolithiasis are high intake of oxalate rich foods, animal protein, less fluid intake, high dietary salt intake. The high percentage of risk factors for each category in our study are less fluid intake: $88 \%$, tomatoes: $86 \%$, ground nuts: $78 \%$, chicken: $60 \%$, high salt intake: $53.1 \%$, mutton: $32.5 \%$, spinach: $26 \%$, black grapes: $16 \%$, beef: $9.3 \%$.

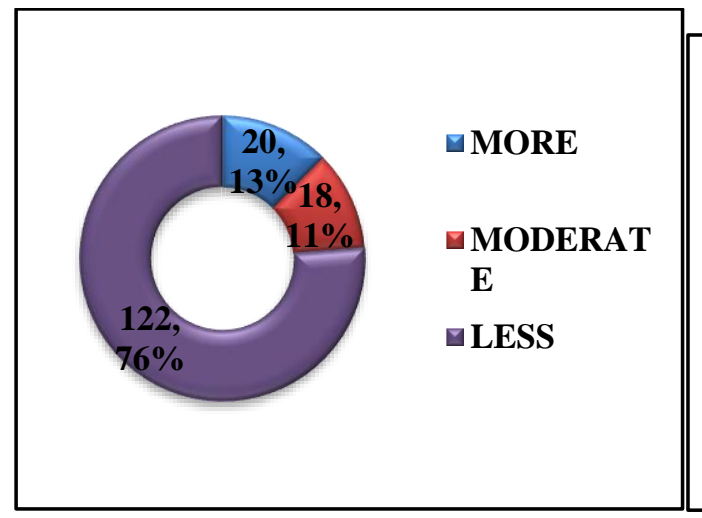

Fig 4 fluid intake

Fig 4 represents the daily fluid intake About $76 \%(\mathrm{n}=122)$ are taking less fluid intake ( $<2$ litres) and $11 \%(\mathrm{n}=18)$ are taking

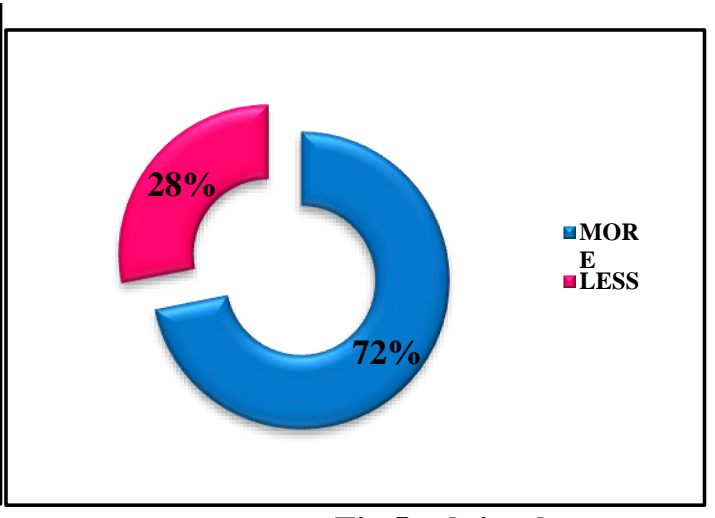

Fig 5 salt intake

moderate fluid intake(2-3 litres) and $13 \%(\mathrm{n}=20)$ are taking more fluid intake (4-5 litres).fig 5 represents salt intake $72 \%$ are of more and $28 \%$ are of less salt intake. 


\begin{tabular}{|l|l|}
\hline Demographic details & $\mathbf{N = 1 6 0}$ \\
\hline Age (mean) & 48.24 \\
Male (\%) & $103(64)$ \\
Females (\%) & $57(36)$ \\
Loin pain(\%) & 53.75 \\
\hline Groin pain(\%) & 13.75 \\
Flank pain(\%) & 23.75 \\
Asymptomatic(\%) & 26 \\
Risk factors & \\
\hline Protein rich foods $(\%)$ & 68.25 \\
\hline
\end{tabular}

Table 1

Table 1 represents the demographic details of patients and clinical symptoms and risk factors. Table 2 represents percentage risk of oxalate rich foods in our study. Of all tomatoes and ground nuts are of more percentage of risk.

\section{DISCUSSION}

In this study the major risk factors are high oxalate intake, high animal protein diet, less fluid intake, more salt intake. We analysed these foods in patients by asking their frequency of use in their daily routine. High oxalate intake is also known as hyperoxaluria. It is the condition triggered by using high intake of oxalate rich foods like tomatoes, spinach, potatoes, sweet potatoes, cashew nuts, black grapes, chocolates, beetroots, bran flakes. By taking these foods in more can cause production of more oxalates in the kidney; these free oxalates conjugate with the free calcium ions which are need to be re-absorbed in the kidney forming calcium-oxalate crystals. These calcium-oxalate crystals aggregate together and form calcium-oxalate stones. Which is the most commonest stones. Intake of calcium rich foods along with oxalate rich foods will be helpful in preventing the increase of size of calculi, as calcium and oxalate get metabolized and absorbed in the intestine. Next about high intake of animal protein which it leads to increased acid production (i.e methionine,cystine,urea,sulphuricacid) thus leads to increase in urinary $\mathrm{P}^{\mathrm{H}}$ which leads to decreased calcium reabsorption further increase of calcium excretion leading to calculi..less fluid intake leads to lead to low urine output. When urine volume is low, the urine can theoretically be easily supersaturated with various solutes, such as calcium, oxalate, phosphorus, and uric acid.increased super saturation leads to crystallization thus forms the first step to stone formation. high intake of sodium leads to increase sodium load thus sodium reabsorption decreases followed by calcium reabsorption thus increases calcium excretion leading to stone formation.

\section{STATISTICAL ANALYSIS}

We used chi square test to analyse the significant relationship between protein and oxalate intake for protein and oxalate intake there is a significant relationship between protein intake and oxalate intake by $p$ value $(\mathrm{p}<0.0001)$ and the chi square value is 7.395 . Individuals with more protein intake are $41.04 \%$
Oxalate rich foods

Tomatoes

Spinach

Potatoes

Beans

Ground nuts

Black grapes

Beetroots
$\mathrm{N}=160(\%)$

85.62

26.87

10

33.75

78.75

16.87

11.87
Table 2

$(n=55)$ and percentage of individuals with more oxalate intake are $58.96 \%(n=79)$.and more salt and less fluid intake..For fluid and salt intake there is a significant relationship between salt intake and fluid intake by $\mathrm{p}$ value $(\mathrm{p}<0.0001)$ and the chi square value is 74.25 Individuals with less fluid intake are $73.05 \%(n=122)$ and percentage of individuals with more salt intake are 75.16 $\%(n=115)$.

\section{Conflict of interest:}

No conflict of interest

\section{CONCLUSION}

Urolithiasis is a most common disease caused by multiple risk factors. Dietary risk factor is one of the noted problem in many urolithiasis patients We observed that treatment of calculi depends on stone size and anatomical position. From our study we conclude that less fluid intake, high oxalate foods, and high animal protein diet, high dietary salt will influence the formation of urolithiasis.

\section{ACKNOWLEDGEMENT}

We wish to express our sincere gratitude to Dr.P Saravana kumar sir, our thesis advisor for the assistance and encouragement to pursue to this study.

\section{REFERENCES}

[1] William.C shiel. jr.mn,facp,facr,medical definition of urolithiasis,27-122018

[2] KN stamatious, VL karanasious,RE lacvoir,NG kavouras, VT papadimitrous,c chlopsios prevalence of urolithiasis,volume 6:610,2006

[3] Gary curhan ,National kidney foundation primer on kidney diseases (sixth edition), 2014

[4] Tahunalelign \& beyene petrus, kidney stones disease update on current concepts, volume 2018,1-12 pages.

[5] Y. I. Rabinovich, M. Esayanur, S. Daosukho, K. J. Byer, H. E. El-Shall, and S. R. Khan, "Adhesion force between calcium oxalate monohydrate crystal and kidney epithelial cells and possible relevance for kidney stone formation," Journal of Colloid and Interface Science, vol. 300, no. 1, pp. 131140, 2006.

[6] C. Türk (Chair), A. Skolarikos (Vice-chair), A. Neisius, A. Petrik, C. Seitz, K. Thomas Guidelines Associates:J.F. Donaldson, T. Drake, N. Grivas, Y. Ruhayeluroweb.org/wp-content/uploads/EAU-Guidelines-on-Urolithiasis2019. 
[7] Haewook Han1*, Adam M. Segal2, Julian L. Seifter3, Johanna T. Dwyer4 Nutritional Management of Kidney Stones (Nephrolithiasis) Clin Nutr Res 2015;4:137-152

\section{AUTHORS}

First Author - Dr.P Saravana kumar, Department of urology, Lalitha super speciality hospital, Guntur Second Author - Gurijala Sai mounika, Department of pharmacy practice, AM Reddy memorial college of pharmacy, Narasaraopet, Guntur (Dst), Andhra Pradesh.
Third Author - Konala raja prabhu sujith, Department of pharmacy practice, AM Reddy memorial college of pharmacy, Narasaraopet, Guntur (Dst), Andhra Pradesh.

Fourth Author - Rajulapati Tejaswini, Department of pharmacy practice, AM Reddy memorial college of pharmacy, Narasaraopet, Guntur (Dst), Andhra Pradesh.

Corresponding address: Projectsts91117@gmail.com 